\title{
Preeclamptic plasma stimulates the expression of miRNAs, leading to a decrease in endothelin-1 production in endothelial cells
}

\author{
Mayara Caldeira-Dias ${ }^{\mathrm{a}}$, Marcelo R. Luizon ${ }^{\mathrm{a}}$, Elenice Deffune ${ }^{\mathrm{b}}$, Jose E. Tanus-Santos ${ }^{\mathrm{c}}$, \\ Paula P. Freire ${ }^{\mathrm{d}}$, Robson F. Carvalho ${ }^{\mathrm{d}}$, Heloisa Bettiol ${ }^{\mathrm{e}}$, Viviane C. Cardoso ${ }^{\mathrm{e}}$, \\ Marco Antonio Barbieri ${ }^{\mathrm{e}}$, Ricardo C. Cavalli ${ }^{\mathrm{f}}$, Valéria C. Sandrim ${ }^{\mathrm{a}, *}$ \\ ${ }^{a}$ Department of Pharmacology, Institute of Biosciences of Botucatu, São Paulo State University (UNESP), Botucatu, SP, Brazil \\ b Blood Transfusion Center, Cell Engineering Laboratory, Botucatu Medical School, São Paulo State University (UNESP), Botucatu, SP, Brazil \\ ${ }^{\mathrm{c}}$ Department of Pharmacology, Ribeirao Preto Medical School, University of Sao Paulo, Ribeirao Preto, SP, Brazil \\ d Department of Morphology, Institute of Biosciences of Botucatu, São Paulo State University (UNESP), Botucatu, SP, Brazil \\ e Department of Pediatrics, Ribeirao Preto Medical School, University of Sao Paulo, Ribeirao Preto, SP, Brazil \\ ${ }_{\mathrm{f}}^{\mathrm{f}}$ Department of Gynecology and Obstetrics, Ribeirao Preto Medical School, University of Sao Paulo, Ribeirao Preto, SP, Brazil
}

\section{A R T I C L E I N F O}

\section{Keywords:}

Endothelial cells

Endothelin-1

miRNAs

Plasma

Preeclampsia

\begin{abstract}
A B S T R A C T
Preeclampsia is a major cause of maternal and fetal morbidity and mortality worldwide. It is a multisystem pregnancy syndrome characterized by general endothelial dysfunction caused mainly by plasma factors and debris in endothelial cells. It is widely accepted that endothelin-1 (ET-1) is involved in the pathophysiology of preeclampsia, and so it is of interest to ascertain whether the ET-1 gene (EDN1) can be targeted with tools such as miRNAs. Therefore, we investigated the relationship between the expression of miRNAs that putatively target EDN1 (and so affect ET-1 levels) in HUVECs incubated with plasma from preeclamptic women. EDN1 expression and ET-1 levels in HUVECs incubated with plasma from women with preeclampsia were similar to those in plasma from healthy pregnant women. Expression of miRNAs let-7a, -7b, and -7c, and to a lesser degree 125a and $125 \mathrm{~b}$, was increased in preeclampsia. Expression of miRNAs of the let-7 family was significantly negatively correlated with ET-1 levels in preeclampsia. Transfection of the preeclampsia cultures with mimic miRNA let-7 decreased ET-1 levels. Our findings show that preeclamptic plasma stimulates the expression of miRNAs in HUVECs, leading to a decrease in ET-1levels, which suggests that therapeutic miRNAs may aid in the management of preeclampsia.
\end{abstract}

\section{Introduction}

Preeclampsia is a multisystem syndrome diagnosed by hypertension after 20 weeks of gestation associated with proteinuria and/or edema $[1,2]$. It affects approximately $2-8 \%$ of all pregnancies and is a major cause of maternal and fetal morbidity and mortality worldwide $[3,4]$. In preeclampsia, the ischemic placental tissue produces and releases factors and debris into the maternal circulation, leading to the general endothelial dysfunction characteristic of the syndrome, which is responsible for its clinical manifestations $[5,6]$.

Through the incubation of endothelial cells with plasma/serum from preeclamptic women, an in vitro model of preeclampsia, this pathophysiological mechanism can be observed [7-16]. Plasma/serum taken from pregnant women who subsequently developed preeclampsia was shown to impair endothelial function in myometrial vessels [7] and to dysregulate $c$-FOS expression in endothelial cells [8]. In addition, as shown by our group and others, plasma/serum taken from women with clinically verified symptoms of preeclampsia induced alterations in endothelial cells [8-12], including changes in endothelin-1 (ET-1) expression $[10,11,14-16]$, a potent vasoconstrictor peptide which has been extensively studied in preeclampsia [17-21].

Recently, it was demonstrated that microRNAs (miRNAs) are involved in the post-transcriptional regulation of the ET-1 gene (EDN1) [22-26]. miRNAs are small (18-24 nucleotides) endogenous noncoding

\footnotetext{
Abbreviations: BMI, body mass index; c-FOS, FOS proto-oncogene; DBP, diastolic blood pressure; EDN1, endothelin-1 gene; ET-1, endothelin-1; GA, gestational age; HEK293A, human

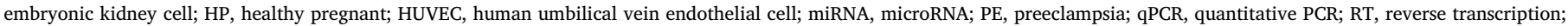
SBP, systolic blood pressure

* Corresponding author at: Department of Pharmacology, Institute of Biosciences of Botucatu, São Paulo State University (UNESP), Botucatu, Distrito Rubiao Junior, SP, Zip-code 18618-000, Brazil.

E-mail address: valeria.sandrim@ibb.unesp.br (V.C. Sandrim).
} 
single-stranded RNAs that post-transcriptionally regulate gene expression by repressing protein expression [27]. Mounting evidence for alterations in miRNA expression has been found in placental tissue and plasma/serum from preeclamptic women, suggesting a role of miRNAs in preeclampsia [28-32]. However, to our knowledge no study has examined the relationship between miRNA expression and ET-1 in endothelial cells incubated with plasma/serum from preeclamptic women.

In the current study, we investigated the relationship between expression of miRNAs that putatively target EDN1 and ET-1 levels in endothelial cells incubated with plasma taken from pregnant women who subsequently developed clinically verified preeclampsia. We also investigated circulating ET-1 levels to verify whether the in vitro results are found systemically.

\section{Materials and methods}

\subsection{Study population}

We recruited two groups of women. One group was derived from a broader observational study in two Brazilian cities, Ribeirão Preto (in the state of São Paulo) and São Luís (in the state of Maranhão), although the women analyzed here came from Ribeirão Preto only. The study was approved by the Institutional Review Board at Ribeirão Preto Medical School, Brazil (reference 4116/2008, approval date November, 11 2008). A total of 1417 pregnant were evaluated at the Hospital das Clínicas Faculdade de Medicina de Ribeirão Preto da Universidade de São Paulo (HCFMRP-USP) at 20-25 weeks of gestation. Of these women, 17 did not return and another 460 gave birth in other hospitals. From the 940 pregnant women remaining, 30 developed preeclampsia and 45 healthy pregnant women were randomly selected as controls. For this study, we included only 29 samples from women who developed preeclampsia, because one sample was too small (in terms of volume) to perform all the experiments.

The other group of women was recruited from the ambulatory clinic at HCFMRP-USP. That study was also approved by the Institutional Review Board at Ribeirão Preto Medical School, Brazil (reference 4682/ 2006, approved date June 20, 2006). This group likewise included both preeclamptic (cases) and healthy women (controls).

'Cases' are those women analyzed before the onset of clinical symptoms, with a corresponding group of 'controls', and the 'PE' group are those women analyzed after the onset of clinical symptoms, with a corresponding 'HP' group of women with a healthy pregnancy throughout (see Fig. S1).

Both studies were performed following the principles of the Declaration of Helsinki. All subjects gave written informed consent. Exclusion criteria included twin pregnancy, hemostatic abnormalities, chronic hypertension, diabetes mellitus, fetal abnormalities, cancer, and cardiovascular, autoimmune, renal, and hepatic diseases. Preeclampsia was characterized as systolic blood pressure (SBP) $\geq 140 \mathrm{mmHg}$ and diastolic blood pressure (DBP) $\geq 90 \mathrm{mmHg}$ on two occasions more than $4 \mathrm{~h}$ apart, occurring after 20 weeks of gestation in a previously normotensive woman, in association with proteinuria ( $\geq 300 \mathrm{mg} / 24-\mathrm{h}$ urine) [2].

All maternal venous blood samples were collected in tubes containing heparin. The tubes were rapidly centrifuged ( $1000 \mathrm{~g}$ for $3 \mathrm{~min}$ ) and plasma samples were stored at $-80^{\circ} \mathrm{C}$.

\section{Endothelial cell culture and plasma incubation}

The human umbilical vein endothelial cell (HUVEC) line (CRL 2873) was obtained from American Type Culture Collection (ATCC) (Manassas, VA, USA). HUVECs were cultured in DMEM medium (Gibco, CA, USA) supplemented with $10 \%(\mathrm{v} / \mathrm{v}$ ) fetal calf serum (FCS) (Gibco), $50 \mu \mathrm{g} / \mathrm{ml}$ penicillin, $50 \mu \mathrm{g} / \mathrm{ml}$ streptomycin and $0.5 \mu \mathrm{g} / \mathrm{ml}$ amphotericin B (Gibco) at $37^{\circ} \mathrm{C}$ in an incubator with a $5 \% \mathrm{CO}_{2}$ atmosphere. Cells were grown in $75-\mathrm{cm}^{3}$ tissue culture flasks (Corning, Costar, Netherlands) and after reaching $80 \%$ confluence, they were detached from the flask surface by trypsin/EDTA $(0.5 / 0.2 \mathrm{mg} / \mathrm{ml}$ in phosphatebuffered saline, PBS) and split at a $1: 3$ ratio into $25-\mathrm{cm}^{3}$ flasks. At passage 3, HUVECs were resuspended in DMEM medium and re-plated in 48-well tissue culture plates (Corning), where they were grown to $80 \%$ confluence for plasma incubation. After reaching confluence, the medium was removed and the cells were washed twice in PBS. The cells were then incubated in medium with $20 \%(\mathrm{v} / \mathrm{v})$ plasma from controls and cases, and from $\mathrm{HP}$ and PE women, for $24 \mathrm{~h}$ at $37^{\circ} \mathrm{C}, 5 \% \mathrm{CO}_{2}$. Cells were used for these experiments until passage 8 .

\section{RNA isolation}

Total RNA from HUVECs incubated with plasma from controls, cases, the HP group and the PE group was isolated using the miRNeasy Mini Kit (Qiagen, Leusden, Netherlands) according to the manufacturer's protocol. Quantification of isolated RNA was performed using a NanoDrop Spectrophotometer (Thermo Scientific, MA, USA), and it was consistently found to be pure.

\section{PCR array analysis for mRNA expression}

$\mathrm{RT}^{2}$ Profiler $^{\mathrm{TM}}$ PCR Array Human Endothelial Cell Biology (Qiagen ${ }^{\circledR}$ PAHS-015ZC) was used to verify the expression of 84 genes related to endothelial cell biology (Table S1), as well as five housekeeping genes. Before gene expression analysis using $\mathrm{RT}^{2}$ Profiler PCR Arrays, the $\mathrm{RT}^{2}$ RNA QC PCR Array (Qiagen ${ }^{\circledR}$ ) was used to assess the quality of isolated RNA, according to the manufacturer's protocol. The array contains controls for RNA integrity, presence of inhibitors of reverse transcription and PCR amplification, and presence of genomic or other DNA contamination. All RNA samples were consistently found to be intact and pure.

For the PCR array, total RNA was converted into cDNA using the $\mathrm{RT}^{2}$ First Strand Kit (Qiagen ${ }^{\circledast}$ ), according to the manufacturer's protocol. qPCR was performed using the $\mathrm{RT}^{2}$ SYBR Green qPCR Mastermix $\left(\right.$ Qiagen $\left.^{\circledR}\right)$, which contained $1350 \mu \mathrm{L}$ of SYBR Green Master Mix, $1248 \mu \mathrm{L}$ of RNase-free water and $102 \mu \mathrm{L}$ of cDNA $(1.5 \mathrm{ng} / \mu \mathrm{L})$ from each sample in a final volume of $2700 \mu \mathrm{L}$ per plate. Thermal cycling was performed under the following conditions: $10 \mathrm{~min}$ at $95^{\circ} \mathrm{C}, 40$ two-step cycles of $15 \mathrm{~s}$ at $95^{\circ} \mathrm{C}$, and $60 \mathrm{~s}$ at $60^{\circ} \mathrm{C}$, and a final step for the dissociation curve. Normalization was performed using the five housekeeping genes (ACTB, B2M, GAPDH, HPRT1, RPLO) in the array. Relative quantification was calculated using the comparative 2(-Delta Delta C(T)) method [33].

\section{Messenger RNA expression by qPCR}

To validate the differential findings regarding the expression of messenger RNA (mRNA) in the PCR array in women after the onset of symptoms of preeclampsia, qPCR was performed using the KiCqStart Universal SYBR Green qPCR (Sigma-Aldrich, Poole, UK). For qPCR, the same isolated RNAs used in the PCR array were used as templates using the High-Capacity cDNA Reverse Transcription Kit (Life Technologies, ON, Canada), according to the manufacturer's protocol. Each reaction contained $10 \mu \mathrm{L}$ KiCqStart SYBR Green qPCR Ready Mix, $300 \mathrm{nM}$ of each primer (forward and reverse), a variable amount of nuclease-free water and $5 \mu \mathrm{L}$ of cDNA $(1.5 \mathrm{ng} / \mu \mathrm{L})$, to give a final volume of $20 \mu \mathrm{L}$.

The following primers were synthesized by Sigma-Aldrich: EDN1, forward 5'CAAGCAGGAAAAGAACTCAG-3' and reverse 5'CTGGTTTGT CTTAGGTGTTC-3'; HPRT1, forward 5'ATAAGCCAGACTTTGTTGG-3' and reverse 5'ATAGGACTCCAGATGTTTCC-3'. The HPRT1 gene was chosen as an endogenous control as it was the gene most stable in the samples from the panel of housekeeping genes in the RT ${ }^{2}$ RNA QC PCR Array (Qiagen ${ }^{\circledast}$ ). Thermal cycling was performed under the following conditions: $10 \mathrm{~min}$ at $95^{\circ} \mathrm{C}, 40$ two-step cycles of $15 \mathrm{~s}$ at $95^{\circ} \mathrm{C}$ and $60 \mathrm{~s}$ 
at $60^{\circ} \mathrm{C}$, and a final step for the dissociation curve for both primers. Relative quantification was calculated using the comparative 2(-Delta Delta C(T)) method [33]. All PCR reactions were performed in duplicate for each sample.

\section{7. miRNA selection}

We initially selected miRNAs to target the mRNA of the EDN1 gene (Table S2) based on bioinformatics analysis using two databases (Microrna.org [34] and miRWalk 2.0 [35]). This selection was refined using two sub-criteria:

1\%2 miRNAs expressed in the human umbilical vein endothelial cell line (HUVEC) [36].

$2 \% 2$ miRNAs associated with preeclampsia $[29,37,38]$.

Based on these criteria, the following miRNAs were selected: let-7a, let-7b, let-7c, miR-1, miR-125a, miR-125b, miR-135a, miR-144 and miR-199.

\section{8. miRNA expression by qPCR}

Reverse transcription (RT) was performed using the miScript II RT Kit (Qiagen ${ }^{\circledast}$ ), according to the manufacturer's protocol. The reaction was performed from total isolated RNA using miScript HiSpec Buffer, which selectively converts mature miRNAs and certain small nucleolar RNAs and small nuclear RNAs into cDNA. For qPCR, the miScript SYBR $^{\circledast}$ Green PCR Kit (Qiagen ${ }^{\circledR}$ ) was used and each reaction contained $10 \mu \mathrm{L}$ of QuantiTect SYBR Green PCR Master Mix, $2 \mu \mathrm{L}$ of universal primer, $2 \mu \mathrm{L}$ of each primer of miRNA, $4 \mu \mathrm{L}$ of nuclease-free water and $2 \mu \mathrm{L}$ of cDNA $(1.5 \mathrm{ng} / \mu \mathrm{L})$, in a final volume of $20 \mu \mathrm{L}$. The primers of the selected miRNAs were obtained from Qiagen ${ }^{\circledast}$. Thermal cycling was performed under the following conditions: $15 \mathrm{~min}$ at $95^{\circ} \mathrm{C}, 40$ threestep cycles of $15 \mathrm{~s}$ at $94^{\circ} \mathrm{C}, 30 \mathrm{~s}$ at $55^{\circ} \mathrm{C}$ and $30 \mathrm{~s}$ at $70^{\circ} \mathrm{C}$, and a final step for the dissociation curve. Relative quantification was calculated using the comparative 2(-Delta Delta C(T)) [33] and normalization was performed to U6 snRNA. In all PCR reactions were performed in duplicate for each sample.

\section{Transfection with miRNA mimics}

HUVECs were seeded at a density of $2 \times 10^{5}$ cells per well in a 6well plate and, after reaching $80 \%$ confluence, transfected with miRNA mimic let-7b or miRNA mimic negative control \#1 (Ambion, CA, USA) $(30 \mathrm{pmol})$ for $12 \mathrm{~h}$, using Lipofectamine ${ }^{\circledR} 2000$ Reagent (Invitrogen, CA, USA) according to the manufacturer's protocol. The mimic negative control was a random miRNA sequence with no known homology to the human transcriptome. After transfection, cells were allowed to incubate for $12 \mathrm{~h}$ at $37^{\circ} \mathrm{C}$ in an incubator with a $5 \% \mathrm{CO}_{2}$ atmosphere, followed by RNA isolation and measurement of ET-1 in the supernatants. Cells were used for these experiments until passage 8 .

\section{Measurement of ET-1 concentrations}

The ET-1 concentrations in the HUVECs culture supernatants and plasma samples were measured using enzyme-linked immunosorbent assay kit Quantikine ${ }^{\circledR}$ ELISA Endothelin-1 (R\&D Systems, MN, USA), according to the manufacturer's protocol.

\subsection{Statistical analysis}

Clinical characteristics and ET-1 levels were compared between preeclampsia groups and their respective controls using t-tests. Pearson's correlation coefficients were used in the correlation analyses. These statistical analyses were performed using GraphPad Prism 5.0 (GraphPad Software, CA, USA). Gene and miRNA expression data were
Table 1

Clinical characteristics of patients used to perform in vitro studies.

\begin{tabular}{lllll}
\hline \multirow{2}{*}{ Parameters } & \multicolumn{3}{l}{ Before } \\
\cline { 2 - 5 } & Controls & Cases & HP & PE \\
\hline N & 6 & 6 & 10 & 14 \\
GA at sampling (weeks) & $23 \pm 2$ & $23 \pm 2$ & $36 \pm 2$ & $35 \pm 3$ \\
Age (years) & $28 \pm 5$ & $28 \pm 4$ & $27 \pm 5$ & $26 \pm 5$ \\
BMI (kg/m ${ }^{2}$ ) & $28 \pm 4$ & $30 \pm 6$ & $30 \pm 4$ & $31 \pm 6$ \\
SBP at sampling (mmHg) & $104 \pm 6$ & $118 \pm 1$ & $113 \pm 7$ & $133 \pm 1^{*}$ \\
DBP at sampling (mmHg) & $64 \pm 4$ & $77 \pm 15$ & $73 \pm 6$ & $85 \pm 8^{*}$ \\
GA at delivery (weeks) & $39 \pm 3$ & $36 \pm 2$ & $39 \pm 2$ & $36 \pm 3^{*}$ \\
\hline
\end{tabular}

Values are the means \pm S.D. or percentage. HP, healthy pregnant; PE, preeclampsia; GA, gestational age; BMI, body mass index; SBP, systolic blood pressure; DBP, diastolic blood pressure. " $\mathrm{P}<0.05$ vs controls.

analyzed using the GeneGlobe Data Analysis Center (Qiagen ${ }^{\circledR}$ ) online platform. For all tests, a $\mathrm{P}$ value $<0.05$ (two-tailed) was considered significant.

\section{Results}

11.1. Effects of plasma incubation on the expression of genes involved in endothelial cell biology in HUVECs

The study workflow is shown in Fig. S1. We compared the plasma samples from pregnant women who subsequently developed preeclampsia (cases) with those from women who remained healthy throughout gestation (controls), and from pregnant women with preeclampsia symptoms established (the PE group) with those from healthy pregnant women (the HP group). First, we compared the effects of plasma incubation on the expression of 84 genes (determined using the PCR array) in HUVECs (Table S1). The clinical characteristics of the women whose plasma samples were collected and used to perform the in vitro studies are shown in Table 1 . As expected, we observed significantly increased systolic blood pressure (SBP) and diastolic blood pressure (DBP) in the PE group compared with the HP group. The gestational age (GA) at delivery was significantly lower for both cases and the PE group compared with their respective controls.

Out of the 84 genes evaluated (Table S1), EDN1 expression was the one that differed most significantly between cases and controls (Table S1; Fig. $1 \mathrm{~A}, \mathrm{p}<0.05$ and fold change $>2.0$ ). In HUVECs incubated with plasma from the PE group compared with HP group, however, there was no significant difference in EDN1 expression between them (Table S1; Fig. 1B, p $<0.05$ and fold change $>2.0$ ). To confirm the PCR array data, we increased the number of samples from the PE and HP groups ( $\mathrm{n}=14$ and $\mathrm{n}=10$, respectively), but EDN1 expression remained not significantly different between the groups $(1.19 \pm 0.22$ vs. $1.02 \pm 0.22$ fold change, respectively) (Fig. S2).

\subsection{Effects of plasma incubation on ET-1 levels in HUVECs}

We examined ET-1 levels in supernatants from the HUVECs incubated with plasma from case and control groups, and from PE and HP groups. ET-1 levels were significantly increased $(\mathrm{p}=0.027)$ in the cases $(1.24 \pm 0.14 \mathrm{pg} / \mathrm{mL})$ compared with controls $(0.79 \pm 0.09 \mathrm{pg} / \mathrm{mL})$ (Fig. 1C). ET-1 levels were similar, though, in the PE and HP groups $(1.68 \pm 0.37$ vs. $1.55 \pm 0.33 \mathrm{pg} / \mathrm{mL}, \quad$ respectively, $\mathrm{p}=0.794)$ (Fig. 1D).

\subsection{Expression of miRNAs that target EDN1 in HUVECs}

We examined whether miRNAs that target EDN1 could modulate ET-1 levels. From the miRNAs selected, four were not expressed under our conditions (miR-1, miR-135a, miR-144 and miR-199). In HUVECS incubated with plasma from the case and control groups, there were no 
A)

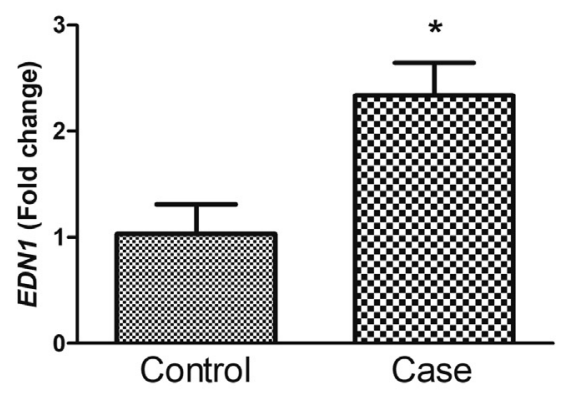

C)

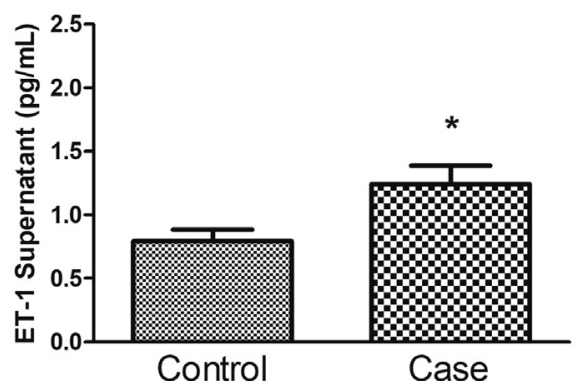

A)

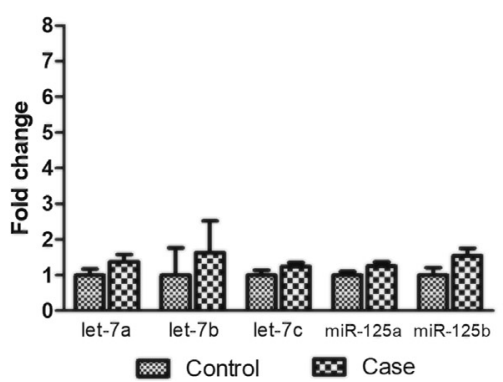

B)

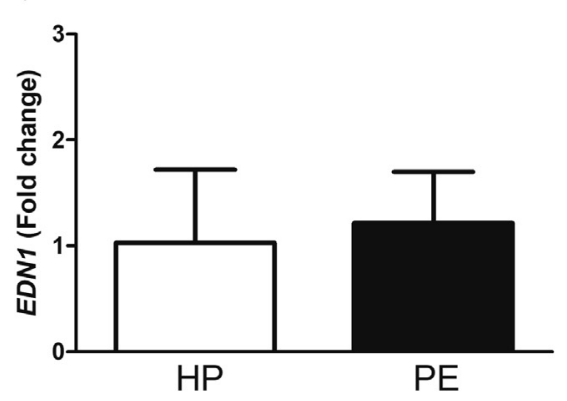

D)

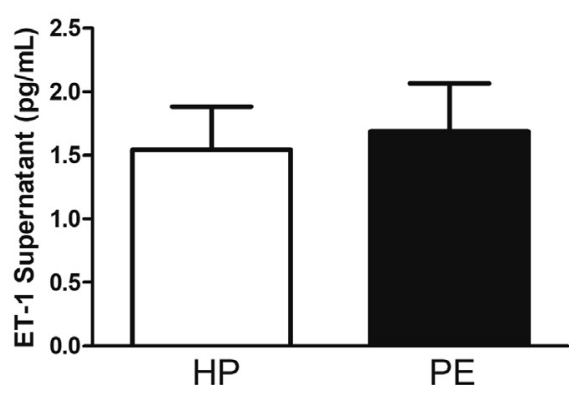

B)

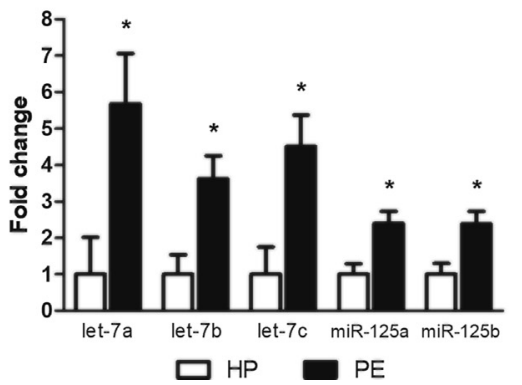

Fig. 1. EDN1 expression and ET-1 levels in HUVECs incubated with plasma from patients before and after clinical symptoms of preeclampsia. HUVECs cells were incubated with $20 \%(\mathrm{v} / \mathrm{v})$ plasma samples from pregnant women who subsequently developed preeclampsia (case) and who remained healthy during gestation (control), and from pregnant women with PE symptoms established (PE) and healthy pregnant women (HP) for $24 \mathrm{~h}$. EDN1 expression by PCR array before (A) and after (B) clinical symptoms of preeclampsia ( $n=6$ per group); ET-1 levels in supernatant by ELISA before (C) and after (D) clinical symptoms of preeclampsia (control and case, $\mathrm{n}=6$; HP, $n=10 ; \quad P E, n=14$ ) (B). Values are fold change \pm S.E.M. relative to respective control (control or HP) (A and B); and means \pm S.E.M. (C and $\mathrm{D}),{ }^{*} \mathrm{P}<0.05$ vs. respective control.

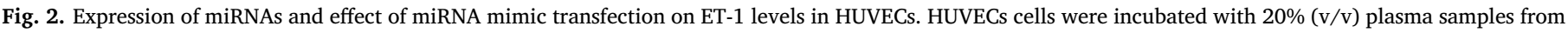

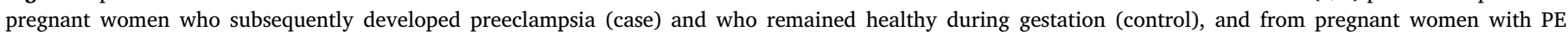

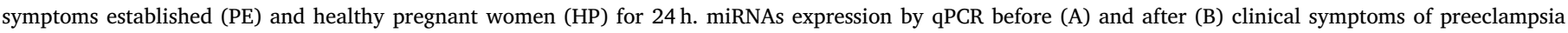

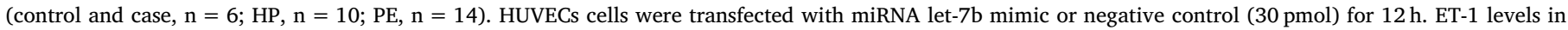

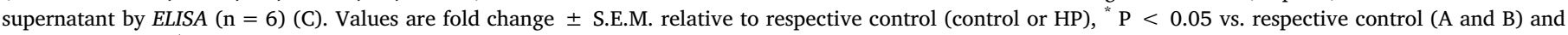
means \pm S.E.M., ${ }^{*} \mathrm{P}<0.05$ vs. negative control (C).

significant differences in miRNA expression between groups (Fig. 2A, $\mathrm{p}>0.05$ and fold change $<2.0$ ). However, in HUVECS incubated with plasma from PE women, miRNAs let-7a, let-7b, let-7c, 125a and $125 \mathrm{~b}$ were significantly upregulated compared with the HP group, and more so for the miRNAs from the let-7 family (Fig. 2B, p $<0.05$ and fold change $>2.0$ ).

Next, we performed correlation analyses on miRNA expression and supernatant ET-1 levels in the PE and HP groups (Table 2).

Table 2

Correlations between Delta $\mathrm{C}_{\mathrm{T}}$ of miRNAs and ET-1 levels (plasma collected after the onset of clinical symptoms of preeclampsia).

\begin{tabular}{lll}
\hline miRNA & HP & PE \\
\hline miR-125a & $-0.15(\mathrm{P}=0.607)$ & $-0.47(\mathrm{P}=0.090)$ \\
miR-125b & $-0.41(\mathrm{P}=0.248)$ & $-0.02(\mathrm{P}=0.910)$ \\
let-7a & $-0.03(\mathrm{P}=0.933)$ & $-0.74(\mathrm{P}=0.003)^{*}$ \\
let-7b & $-0.01(\mathrm{P}=0.932)$ & $-0.76(\mathrm{P}=0.002)^{*}$ \\
let-7c & $-0.06(\mathrm{P}=0.838)$ & $-0.78(\mathrm{P}=0.001)^{*}$
\end{tabular}

Values are Pearson Correlation Coefficient (P-value). HP, healthy pregnant; PE, preeclampsia. " $\mathrm{P}<0.05$.
Interestingly, we found significant inverse correlations between expression of the miRNAs in the let-7 family (a, b and c) and ET-1 levels in the PE group, but not in the HP group.

\subsection{Effect of miRNA mimic let-7b transfection on ET-1 levels in HUVECs}

In order to validate the interaction between miRNAs of the let-7 family and ET-1 levels, suggested by the significant inverse correlations, we evaluated the effect of miRNA mimic let-7b transfection on ET-1 levels in the HUVEC supernatants. Mimic let-7b transfection significantly decreased ET- 1 levels by approximately $25 \%$ compared with the negative control $(3.94 \pm 0.38$ vs. $5.22 \pm 0.14 \mathrm{pg} / \mathrm{mL}$, respectively, $\mathrm{p}=0.010$ ) (Fig. $2 \mathrm{C}$ ). Transfection with the miRNA mimic was confirmed using real-time PCR (qPCR) (Fig. S3).

\subsection{ET-1 levels in plasma from patients before and after the onset of} preeclampsia symptoms and from controls who had healthy pregnancies

Finally, we increased the sample size and quantified plasma ET-1 levels in all the study groups. The clinical characteristics of each group 
Table 3

Clinical characteristics of patients used to quantify circulating ET-1 levels.

\begin{tabular}{|c|c|c|c|c|}
\hline \multirow[t]{2}{*}{ Parameters } & \multicolumn{2}{|l|}{ Before } & \multicolumn{2}{|l|}{ After } \\
\hline & Control & Case & HP & $\mathrm{PE}$ \\
\hline $\mathrm{N}$ & 45 & 29 & 26 & 37 \\
\hline $\begin{array}{l}\text { GA at sampling } \\
\text { (weeks) }\end{array}$ & $24 \pm 2$ & $23 \pm 1$ & $37 \pm 2$ & $35 \pm 3$ \\
\hline Age (years) & $27 \pm 6$ & $28 \pm 6$ & $24 \pm 5$ & $28 \pm 7^{*}$ \\
\hline BMI $\left(\mathrm{kg} / \mathrm{m}^{2}\right)$ & $29 \pm 4$ & $31 \pm 6$ & $31 \pm 6$ & $31 \pm 7$ \\
\hline $\begin{array}{l}\text { SBP at sampling } \\
(\mathrm{mmHg})\end{array}$ & $110 \pm 10$ & $117 \pm 12^{*}$ & $113 \pm 9$ & $140 \pm 13^{*}$ \\
\hline $\begin{array}{c}\text { DBP at sampling } \\
(\mathrm{mmHg})\end{array}$ & $66 \pm 6$ & $74 \pm 9^{*}$ & $71 \pm 7$ & $86 \pm 10^{*}$ \\
\hline Nulliparous (\%) & 36 & 38 & 58 & 46 \\
\hline $\begin{array}{l}\text { GA at delivery } \\
\text { (weeks) }\end{array}$ & $39 \pm 2$ & $36 \pm 4^{*}$ & $40 \pm 1$ & $37 \pm 3^{*}$ \\
\hline $\begin{array}{l}\text { Newborn weight } \\
\text { (g) }\end{array}$ & $3368 \pm 467$ & $2793 \pm 987^{*}$ & $3302 \pm 385$ & $2854 \pm 882^{*}$ \\
\hline
\end{tabular}

Values are the means \pm S.D. or percentage. HP, healthy pregnant; PE, preeclampsia; GA, gestational age; BMI, body mass index; SBP, systolic blood pressure; DBP, diastolic blood pressure. " $\mathrm{P}<0.05$ vs. controls.

are shown in Table 3. As expected, both cases and the PE group had significantly higher SBP and DBP than the controls. Moreover, both preeclampsia groups (cases and PE) had a lower GA at delivery and lower newborn weight compared with the respective control groups.

We found higher ET-1 levels in the cases compared with controls $(0.75 \pm 0.05$ vs. $0.61 \pm 0.03 \mathrm{pg} / \mathrm{mL}$, respectively, $\mathrm{p}=0.023)$ (Fig. 3A) and in the PE group compared with the HP group $(1.53 \pm 0.17$ vs. $1.11 \pm 0.08 \mathrm{pg} / \mathrm{mL}$, respectively, $\mathrm{p}=0.040)$ (Fig. 3B).

\section{Discussion}

In this study, we found increased expression of miRNAs that putatively target EDN1 in HUVECs incubated with plasma from the PE group, compared with plasma from the HP group. The same was not found before the clinical onset of preeclampsia. In addition, we observed significant inverse correlations between miRNA expression of the let-7 family and ET-1 levels in the PE group, but not in the HP group. Therefore, as EDN1 expression and ET-1 levels in the supernatant was increased in cases compared with controls and were not different in the PE group compared with the HP group, we suggest that miRNAs may modulate ET-1 levels in HUVECs in the presence of preeclamptic plasma.

It is widely accepted that ET-1 is involved in the pathophysiology of preeclampsia [17-21], and so it is interest to ascertain whether the ET-1 gene can be targeted with tools such as miRNAs. The seed sequence predicted in which miRNAs let-7 a, b and c bind to EDN1 3'UTR is CUACCUC (positions 572-579). In order to verify the interaction between these miRNAs and the EDN1 gene, we transfected HUVECs with miRNA mimic let-7 and found a significant decrease in ET-1 levels compared with the miRNA negative control condition. Furthermore, the interaction between miRNAs and EDN1 was confirmed by luciferase reporter assays [25,26]. The let-7c inhibited luciferase activity by $>50 \%$, and let- $7 \mathrm{~b}$ also slightly suppressed the luciferase activity in gastric cancer cells [25]. Moreover, the interaction between miR-125a, miR-125b and EDN1 has also been reported in human embryonic kidney cells (HEK293A) [26]. These functional studies validated the interaction between these miRNAs and EDN1, further supporting our hypothesis that these miRNAs modulate ET-1 levels.

The mechanism by which plasma from preeclamptic women stimulates miRNA expression in endothelial cells needs further investigation. It could be through alterations at the transcriptional level or at the post-transcriptional level. At the transcriptional level, it could be changes in the expression of host genes where the miRNA is encoded (for intragenic miRNAs), in transcription factors (for intergenic miRNAs) or in methylation of the promoter in both intragenic and intergenic miRNAs [39]. At the post-transcriptional level, changes in miRNA expression could be due to alterations in miRNA biogenesis, for example in the activity of miRNA biogenesis enzymes such as Dicer and Drosha [39].

Discordant findings have been reported regarding the effects of plasma (collected after the onset of preeclampsia) from preeclamptic women on ET-1 levels in endothelial cells [10,11,14-16]. Increased production of ET-1 was reported in endothelial cells incubated for $24 \mathrm{~h}$ with serum $(20 \%, \mathrm{v} / \mathrm{v})$ from preeclamptic women compared with serum from women with a normal pregnancy [10,11]. Conversely, other studies showed decreased ET-1 levels $[14,16]$ or similar levels [15], which is in agreement with our findings. However, it is important to note that these controversial findings may have resulted from differences in experimental protocols, such as: the plasma/serum concentration during incubation; the time of incubation and proliferation; and the viability and type of HUVEC used (primary or immortalized cell line). Moreover, according to our findings, miRNAs that target EDN1, mainly the let-7b family, may decrease ET-1 levels in HUVECs incubated with plasma from preeclamptic patients.

Many studies have examined the therapeutic potential of miRNAs for pathologies such as cancer, diabetes and cardiovascular diseases, but few miRNAs are in the pre-clinical or clinical stage of development as therapeutic agents $[40,41]$. We found that transfection with miRNA mimic let-7 decreased ET-1 levels in HUVECs, which suggests that miRNA mimics of let-7 might be useful candidates for therapeutic intervention in the management of preeclampsia. However, more studies should be performed to explore this possibility.

To our knowledge, the only study regarding ET-1 levels in plasma samples from pregnant women collected before the onset of symptoms of preeclampsia found higher ET-1 levels in samples from women who subsequently developed mild to severe preeclampsia and eclampsia [42]. This result is in agreement with our findings and, taken together with our in vitro findings, suggests that ET-1 may be a useful biomarker to help identify women at risk of preeclampsia. Furthermore, ET-1 has been validated to predict cardiovascular risk [43]. In pregnant women who had already developed preeclampsia, plasma ET-1 levels were also
A)

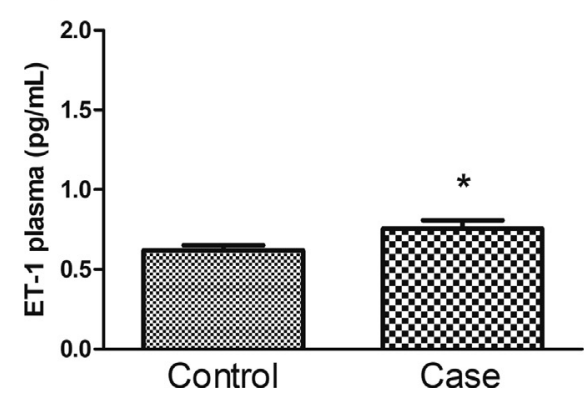

B)

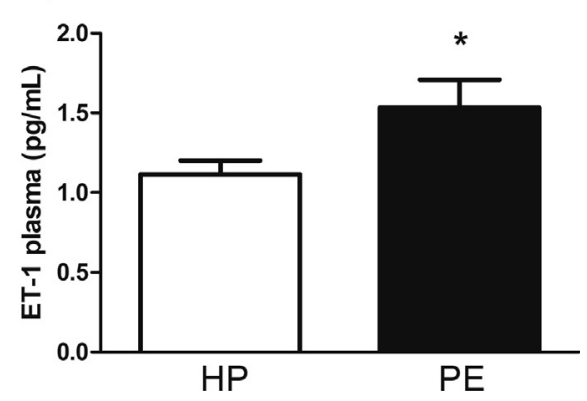

Fig. 3. ET-1 levels in plasma from patients before and after clinical symptoms of preeclampsia. Plasma samples were collected from pregnant women who subsequently developed preeclampsia (case) and who remained healthy during gestation (control), and from pregnant women with PE symptoms established (PE) and healthy pregnant women (HP). ET-1 levels in plasma by ELISA before (A) and after (B) clinical symptoms of preeclampsia (control, $\mathrm{n}=45$; case, $\mathrm{n}=29 ; \quad \mathrm{HP}, \mathrm{n}=26 ; \mathrm{PE}, \mathrm{n}=37)$. Values are means \pm S.E.M., " $\mathrm{P}<0.05$ vs. respective control. 
higher compared with those of healthy pregnant women, which has been extensively observed in previous studies of preeclampsia $[17,18,21]$. This shows that the suppression of ET-1 levels by miRNAs in our in vitro findings occurs locally, not systemically, in preeclamptic women. Moreover, as a limitation of the study, we used an immortalized cell line of HUVECs, which is a healthy cell line; however, it is well established that the systemic endothelial dysfunction characteristic of preeclampsia occurs because the maternal endothelium is already impaired [44].

\section{Conclusions}

In conclusion, we have shown that plasma from preeclamptic women stimulates the expression of miRNAs, mainly miRNAs of the let7 family, leading to a decrease in ET-1 production in HUVECs. This suggests that miRNAs may be a therapeutic tool in the management of preeclampsia.

\section{Acknowledgements}

All authors thank Helio Kushima for technical assistance in ELISA experiments and Ana Lívia de Carvalho Bovolato for assistance in HUVEC culture and plasma incubation.

\section{Conflict of interest}

None.

Funding

This work was supported by the Conselho Nacional de Desenvolvimento Científico e Tecnológico (CNPq-Brazil) [Grant Numbers 131125/2014-1, 305587/2014-5] and by the Fundação de Amparo à Pesquisa do Estado de São Paulo (FAPESP-Brazil) [Grant Number 2013/12919-9].

\section{Appendix A. Supplementary data}

Supplementary data associated with this article can be found, in the online version, at https://doi.org/10.1016/j.preghy.2018.03.001.

\section{References}

[1] B. Sibai, G. Dekker, M. Kupferminc, Pre-eclampsia, Lancet 365 (2005) 785-799.

[2] M.A. Brown, M.D. Lindheimer, M. de Swiet, A. Van Assche, J.-M. Moutquin, The classification and diagnosis of the hypertensive disorders of pregnancy: statement from the international society for the study of hypertension in pregnancy (ISSHP), Hypertens. Pregnancy 20 (2001) ix-xiv.

[3] L. Duley, The global impact of pre-eclampsia and eclampsia, Semin. Perinatol. 33 (2009) 130-137.

[4] J.A. Hutcheon, S. Lisonkova, K.S. Joseph, Epidemiology of pre-eclampsia and the other hypertensive disorders of pregnancy, Best Pract. Res. Clin. Obstet. Gynaecol. 25 (2011) 391-403.

[5] J.M. Roberts, Preeclampsia: what we know and what we do not know, Semin. Perinatol. 24 (2000) 24-28.

[6] C.E. Powe, R.J. Levine, S.A. Karumanchi, Preeclampsia, a disease of the maternal endothelium: the role of antiangiogenic factors and implications for later cardiovascular disease, Circulation 123 (2011) 2856-2869.

[7] J. Myers, G. Mires, M. Macleod, P. Baker, In preeclampsia, the circulating factors capable of altering in vitro endothelial function precede clinical disease, Hypertension 45 (2005) 258-263.

[8] R.M. MacKenzie, V.C. Sandrim, D.M. Carty, J.D. McClure, D.J. Freeman, A.F. Dominiczak, M.W. McBride, C. Delles, Endothelial FOS expression and preeclampsia, BJOG 119 (2012) 1564-1571.

[9] R. Calicchio, C. Buffat, J.R. Mathieu, N. Ben Salem, C. Mehats, S. Jacques, A. Hertig, N. Berkane, J. Grevoul-Fresquet, U. Simeoni, C. Peyssonnaux, J. Gavard, D. Vaiman, F. Miralles, Preeclamptic plasma induces transcription modifications involving the AP-1 transcriptional regulator JDP2 in endothelial cells, Am. J. Pathol. 183 (2013) 1993-2006.

[10] F. Scalera, D. Schlembach, E. Beinder, Production of vasoactive substances by human umbilical vein endothelial cells after incubation with serum from preeclamptic patients, Eur. J. Obstet. Gynecol. Reprod. Biol. 99 (2001) 172-178.
[11] L.V. Kiprono, K. Wallace, J. Moseley, J. Martin, B. LaMarca, Progesterone blunts vascular endothelial cell secretion of endothelin-1 in response to placental ischemia, Am. J. Obstet. Gynecol. 209 (2013) 44.e1-44.e6.

[12] V.C. Sandrim, M.C. Dias, A.L. de C. Bovolato, J.E. Tanus-Santos, E. Deffune, R.C. Cavalli, Plasma from pre-eclamptic patients induces the expression of the antiangiogenic miR-195-5p in endothelial cells, J. Cell. Mol. Med. 20 (2016) 1198-1200.

[13] M.R. Luizon, M. Caldeira-Dias, E. Deffune, K.S. Fernandes, R.C. Cavalli, J.E. Tanus Santos, V.C. Sandrim, Antihypertensive therapy in pre-eclampsia: effects of plasma from nonresponsive patients on endothelial gene expression, Pharmacogenomics (2016).

[14] V.C. Zammit, J.A. Whitworth, M.A. Brown, Endothelium-derived prostacyclin: effect of serum from women with normal and hypertensive pregnancy, Clin. Sci. (Lond) 82 (1992) 383-388.

[15] E.D. Gallery, J. Rowe, S. Campbell, T. Hawkins, Effect of serum on secretion of prostacyclin and endothelin-1 by decidual endothelial cells from normal and preeclamptic pregnancies, Am. J. Obstet. Gynecol. 173 (1995) 918-923.

[16] D.W. Branch, D.J. Dudley, M.D. Mitchell, Preliminary evidence for homoeostatic mechanism regulating endothelin production in pre-eclampsia, Lancet (London, England) 337 (1991) 943-945.

[17] S. Aydin, A. Benian, R. Madazli, S. Uludag, H. Uzun, S. Kaya, Plasma malondialdehyde, superoxide dismutase, sE-selectin, fibronectin, endothelin-1 and nitric oxide levels in women with preeclampsia, Eur. J. Obstet. Gynecol. Reprod. Biol. 113 (2004) 21-25.

[18] F. Bernardi, L. Constantino, R. MacHado, F. Petronilho, F. Dal-Pizzol, Plasma nitric oxide, endothelin-1, arginase and superoxide dismutase in pre-eclamptic women, J. Obstet. Gynaecol. Res. 34 (2008) 957-963.

[19] K. Verdonk, L. Saleh, S. Lankhorst, J.E.I. Smilde, M.M. van Ingen, I.M. Garrelds, E.C.H. Friesema, H. Russcher, A.H. van den Meiracker, W. Visser, A.H.J. Danser, Association studies suggest a key role for endothelin-1 in the pathogenesis of preeclampsia and the accompanying renin-angiotensin-aldosterone system suppression, Hypertension 65 (2015) 1316-1323.

[20] A. Jain, M. Olovsson, G.J. Burton, H. Yung, Endothelin-1 induces endoplasmic reticulum stress by activating the PLC-IP(3) pathway: implications for placental pathophysiology in preeclampsia, Am. J. Pathol. 180 (2012) 2309-2320.

[21] F.C. Bernardi, F. Vuolo, F. Petronilho, M. Michels, C. Ritter, F. Dal-Pizzol, Plasma nitric oxide, endothelin-1, arginase and superoxide dismutase in the plasma and placentae from preeclamptic patients, An. Acad. Bras. Cienc. 87 (2015) 713-719.

[22] M.E. Jacobs, C.S. Wingo, B.D. Cain, An emerging role for microRNA in the regulation of endothelin-1, Front. Physiol. 4 (2013) 22.

[23] B. Feng, Y. Cao, S. Chen, M. Ruiz, S. Chakrabarti, MiRNA-1 regulates endothelin-1 in diabetes, Life Sci. 98 (2014) 18-23.

[24] C. Li, C.S. Gonsalves, M.-S. Eiymo, Mwa Mpollo, P. Malik, S.M. Tahara, V.K. Kalra, MicroRNA 648 Targets ET-1 mRNA and is cotranscriptionally regulated with MICAL3 by PAX5, Mol. Cell. Biol. 35 (2015) 514-528.

[25] K.-W. Tsai, L.-Y. Hu, T.-W. Chen, S.-C. Li, M.-R. Ho, S.-Y. Yu, Y.-T. Tu, W.-S. Chen, H.-C. Lam, Emerging role of microRNAs in modulating endothelin-1 expression in gastric cancer, Oncol. Rep. 33 (2015) 485-493.

[26] D. Li, P. Yang, Q. Xiong, X. Song, X. Yang, L. Liu, W. Yuan, Y.-C. Rui, MicroRNA$125 \mathrm{a} / \mathrm{b}-5 \mathrm{p}$ inhibits endothelin-1 expression in vascular endothelial cells, J. Hypertens. 28 (2010) 1646-1654.

[27] D.P. Bartel, MicroRNAs: target recognition and regulatory functions, Cell 136 (2009) 215-233.

[28] R. Luo, X. Shao, P. Xu, Y. Liu, Y. Wang, Y. Zhao, M. Liu, L. Ji, Y.-X. Li, C. Chang, J. Qiao, C. Peng, Y.-L. Wang, MicroRNA-210 contributes to preeclampsia by downregulating potassium channel modulatory factor 1, Hypertension 64 (2014) 839-845.

[29] L. Wu, H. Zhou, H. Lin, J. Qi, C. Zhu, Z. Gao, H. Wang, Circulating microRNAs are elevated in plasma from severe preeclamptic pregnancies, Reproduction 143 (2012) 389-397.

[30] F. Jiang, J. Li, G. Wu, Z. Miao, L. Lu, G. Ren, X. Wang, Upregulation of microRNA335 and microRNA-584 contributes to the pathogenesis of severe preeclampsia through downregulation of endothelial nitric oxide synthase, Mol. Med. Rep. (2015).

[31] V.C. Sandrim, N. Eleuterio, E. Pilan, J.E. Tanus-Santos, K. Fernandes, R. Cavalli, Plasma levels of increased miR-195-5p correlates with the sFLT-1 levels in preeclampsia, Hypertens. Pregnancy 35 (2016) 150-158.

[32] V.C. Sandrim, M.R. Luizon, A.C. Palei, J.E. Tanus-Santos, R.C. Cavalli, Circulating microRNA expression profiles in pre-eclampsia: evidence of increased miR-885-5p levels, BJOG (2016).

[33] K.J. Livak, T.D. Schmittgen, Analysis of relative gene expression data using real time quantitative PCR and the 2(-Delta Delta C(T)) Method, Methods 25 (2001) 402-408.

[34] D. Betel, M. Wilson, A. Gabow, D.S. Marks, C. Sander, The microRNA.org resource: targets and expression, Nucl. Acids Res. 36 (2008) D149-D153.

[35] H. Dweep, N. Gretz, miRWalk2.0: a comprehensive atlas of microRNA-target interactions, Nat. Methods 12 (2015) 697.

[36] M.N. McCall, O.A. Kent, J. Yu, K. Fox-Talbot, A.L. Zaiman, M.K. Halushka, MicroRNA profiling of diverse endothelial cell types, BMC Med. Genomics 4 (2011) 78.

[37] P. Xu, Y. Zhao, M. Liu, Y. Wang, H. Wang, Y.X. Li, X. Zhu, Y. Yao, H. Wang, J. Qiao, L. Ji, Y.L. Wang, Variations of microRNAs in human placentas and plasma from preeclamptic pregnancy, Hypertension 63 (2014) 1276-1284.

[38] H. Li, Q. Ge, L. Guo, Z. Lu, Maternal plasma miRNAs expression in preeclamptic pregnancies, Biomed. Res. Int. 2013 (2013).

[39] L.F. Gulyaeva, N.E. Kushlinskiy, Regulatory mechanisms of microRNA expression, 
J. Transl. Med. 1-10 (2016).

[40] R. Rupaimoole, F.J. Slack, MicroRNA therapeutics: towards a new era for the management of cancer and other diseases, Nat. Rev. Drug Discov. 16 (2017) 203-222.

[41] B.D. Adams, C. Parsons, L. Walker, W.C. Zhang, F.J. Slack, Targeting noncoding RNAs in disease, J. Clin. Invest. 127 (2017) 761-771.
[42] M. Shaarawy, A.M. Abdel-Magid, Plasma endothelin-1 and mean arterial pressure in the prediction of pre-eclampsia, Int. J. Gynaecol. Obstet. 68 (2000) 105-111.

[43] J. Wang, G.-J. Tan, L.-N. Han, Y.-Y. Bai, M. He, H.-B. Liu, Novel biomarkers for cardiovascular risk prediction, J. Geriatr. Cardiol. 14 (2017) 135-150.

[44] J. Roberts, Endothelial Dysfunction in Preeclampsia, Semin. Reprod. Med. 16 (1998) 5-15. 